\title{
First Directional Measurement of Sub-MeV Solar Neutrinos with Borexino
}

M. Agostini, ${ }^{25,16}$ K. Altenmüller, ${ }^{16}$ S. Appel, ${ }^{16}$ V. Atroshchenko, ${ }^{6}$ Z. Bagdasarian, ${ }^{22,{ }^{*}}$ D. Basilico, ${ }^{9}$ G. Bellini, ${ }^{9}$ J. Benziger, ${ }^{13}$ R. Biondi, ${ }^{8}$ D. Bravo,${ }^{9, \$}$ B. Caccianiga, ${ }^{9}$ F. Calaprice, ${ }^{12}$ A. Caminata, ${ }^{3}$ P. Cavalcante, ${ }^{15}$ A. Chepurnov, ${ }^{17}$ D. D'Angelo, ${ }^{9}$ S. Davini, ${ }^{3}$ A. Derbin, ${ }^{11}$ A. Di Giacinto, ${ }^{8}$ V. Di Marcello, ${ }^{8}$ X. F. Ding, ${ }^{12}$ A. Di Ludovico, ${ }^{12}$ L. Di Noto, ${ }^{3}$ I. Drachnev, ${ }^{11}$ A. Formozov, ${ }^{2,9}$ D. Franco, ${ }^{1}$ C. Galbiati, ${ }^{12,18}$ C. Ghiano, ${ }^{8}$ M. Giammarchi, ${ }^{9}$ A. Goretti, ${ }^{12,4,8}$ A. S. Göttel,,${ }^{22,23}$ M. Gromov, ${ }^{17,2}$ D. Guffanti, ${ }^{20}$ Aldo Ianni, ${ }^{8}$ Andrea Ianni, ${ }^{12}$ A. Jany, ${ }^{4}$ D. Jeschke, ${ }^{16}$ V. Kobychev, ${ }^{5}$ G. Korga, ${ }^{24,26}$ S. Kumaran, ${ }^{22,23}$ M. Laubenstein, ${ }^{8}$ E. Litvinovich, ${ }^{6,7}$ P. Lombardi, ${ }^{9}$ I. Lomskaya, ${ }^{11}$ L. Ludhova, ${ }^{22,23}$ G. Lukyanchenko, ${ }^{6}$ L. Lukyanchenko, ${ }^{6}$ I. Machulin,,${ }^{6,7}$ J. Martyn $\odot,{ }^{20}$ E. Meroni,${ }^{9}$ M. Meyer, ${ }^{19}$ L. Miramonti, ${ }^{9}$ M. Misiaszek, ${ }^{4}$ V. Muratova, ${ }^{11}$ B. Neumair ${ }^{16}$ M. Nieslony, ${ }^{20}$ R. Nugmanov ${ }^{6,7}$ L. Oberauer, ${ }^{16}$ V. Orekhov, ${ }^{20}$ F. Ortica, ${ }^{10}$ M. Pallavicini, ${ }^{3}$ L. Papp, ${ }^{16}$ L. Pelicci, ${ }^{22,23}$ Ö. Penek, ${ }^{22}$ L. Pietrofaccia, ${ }^{12}$ N. Pilipenko, ${ }^{11}$ A. Pocar, ${ }^{14}$ G. Raikov, ${ }^{6}$ M. T. Ranalli, ${ }^{8}$ G. Ranucci, ${ }^{9}$ A. Razeto, ${ }^{8}$ A. Re,${ }^{9}$ M. Redchuk,${ }^{22,23, \|}$ A. Romani, ${ }^{10}$ N. Rossi, ${ }^{8}$ S. Schönert, ${ }^{16}$ D. Semenov, ${ }^{11}$ G. Settanta, ${ }^{22,}{ }^{\prime}$ M. Skorokhvatov, ${ }^{6,7}$ A. Singhal, ${ }^{22,23}$ O. Smirnov, ${ }^{2}$ A. Sotnikov, ${ }^{2}$ Y. Suvorov, ${ }^{8,6,}$ R. Tartaglia, ${ }^{8}$ G. Testera, ${ }^{3}$ J. Thurn, ${ }^{19}$ E. Unzhakov, ${ }^{11}$ A. Vishneva, ${ }^{2}$ R. B. Vogelaar ${ }^{15}$ F. von Feilitzsch, ${ }^{16}$ A. Wessel, ${ }^{21,22,23}$ M. Wojcik, ${ }^{4}$ B. Wonsak, ${ }^{27}$ M. Wurm, ${ }^{20}$ S. Zavatarelli, ${ }^{3}$ K. Zuber, ${ }^{19}$ and G. Zuzel ${ }^{4}$

(Borexino Collaboration) $^{* *}$

\author{
${ }^{1}$ APC, Université de Paris, CNRS, Astroparticule et Cosmologie, Paris F-75013, France \\ ${ }^{2}$ Joint Institute for Nuclear Research, 141980 Dubna, Russia \\ ${ }^{3}$ Dipartimento di Fisica, Università degli Studi e INFN, 16146 Genova, Italy \\ ${ }^{4}$ M. Smoluchowski Institute of Physics, Jagiellonian University, 30348 Krakow, Poland \\ ${ }^{5}$ Institute for Nuclear Research of NAS Ukraine, 03028 Kyiv, Ukraine \\ ${ }^{6}$ National Research Centre Kurchatov Institute, 123182 Moscow, Russia \\ ${ }^{7}$ National Research Nuclear University MEPhI (Moscow Engineering Physics Institute), 115409 Moscow, Russia \\ ${ }^{8}$ INFN Laboratori Nazionali del Gran Sasso, 67010 Assergi (AQ), Italy \\ ${ }^{9}$ Dipartimento di Fisica, Università degli Studi e INFN, 20133 Milano, Italy \\ ${ }^{10}$ Dipartimento di Chimica, Biologia e Biotecnologie, Università degli Studi e INFN, 06123 Perugia, Italy \\ ${ }^{11}$ St. Petersburg Nuclear Physics Institute NRC Kurchatov Institute, 188350 Gatchina, Russia \\ ${ }^{12}$ Physics Department, Princeton University, Princeton, New Jersey 08544, USA \\ ${ }^{13}$ Chemical Engineering Department, Princeton University, Princeton, New Jersey 08544, USA \\ ${ }^{14}$ Amherst Center for Fundamental Interactions and Physics Department, UMass, Amherst, Massachusetts 01003, USA \\ ${ }^{15}$ Physics Department, Virginia Polytechnic Institute and State University, Blacksburg, Virginia 24061, USA \\ ${ }^{16}$ Physik-Department, Technische Universität München, 85748 Garching, Germany \\ ${ }^{17}$ Lomonosov Moscow State University Skobeltsyn Institute of Nuclear Physics, 119234 Moscow, Russia \\ ${ }^{18}$ Gran Sasso Science Institute, 67100 L'Aquila, Italy \\ ${ }^{19}$ Department of Physics, Technische Universität Dresden, 01062 Dresden, Germany \\ ${ }^{20}$ Institute of Physics and Cluster of Excellence PRISMA+, Johannes Gutenberg-Universität Mainz, 55099 Mainz, Germany \\ ${ }^{21}$ GSI Helmholtzzentrum für Schwerionenforschung, Planckstrasse 1, D-64291 Darmstadt, Germany \\ ${ }^{22}$ Institut für Kernphysik, Forschungszentrum Jülich, 52425 Jülich, Germany \\ ${ }^{23}$ III. Physikalisches Institut B, RWTH Aachen University, 52062 Aachen, Germany \\ ${ }^{24}$ Department of Physics, School of Engineering, Physical and Mathematical Sciences, Royal Holloway, University of London, \\ Egham, TW20 OEX, United Kingdom \\ ${ }^{25}$ Department of Physics and Astronomy, University College London, London, WC1E 6BT, United Kingdom \\ ${ }^{26}$ Institute of Nuclear Research (Atomki), 4026, Debrecen, Hungary \\ ${ }^{27}$ University of Hamburg, Institute of Experimental Physics, Luruper Chaussee 149, 22761 Hamburg, Germany
}

(Received 22 December 2021; accepted 25 January 2022; published 3 March 2022)

\begin{abstract}
Published by the American Physical Society under the terms of the Creative Commons Attribution 4.0 International license. Further distribution of this work must maintain attribution to the author(s) and the published article's title, journal citation, and DOI. Funded by $S C O A P^{3}$.
\end{abstract}


We report the measurement of sub-MeV solar neutrinos through the use of their associated Cherenkov radiation, performed with the Borexino detector at the Laboratori Nazionali del Gran Sasso. The measurement is achieved using a novel technique that correlates individual photon hits of events to the known position of the Sun. In an energy window between 0.54 to $0.74 \mathrm{MeV}$, selected using the dominant scintillation light, we have measured $10887_{-2103}^{+2386}$ (stat) \pm 947 (syst) (68\% confidence interval) solar neutrinos out of 19904 total events. This corresponds to a ${ }^{7} \mathrm{Be}$ neutrino interaction rate of $51.6_{-12.5}^{+13.9}$ counts/(day $\cdot 100$ ton), which is in agreement with the standard solar model predictions and the previous spectroscopic results of Borexino. The no-neutrino hypothesis can be excluded with $>5 \sigma$ confidence level. For the first time, we have demonstrated the possibility of utilizing the directional Cherenkov information for sub-MeV solar neutrinos, in a large-scale, high light yield liquid scintillator detector. This measurement provides an experimental proof of principle for future hybrid event reconstruction using both Cherenkov and scintillation signatures simultaneously.

DOI: 10.1103/PhysRevLett.128.091803

Introduction.-Because of their tiny interaction cross sections, neutrinos provide us with unique information about otherwise inaccessible locations such as the center of stars and other astronomical objects. Solar neutrinos are created as electron-flavored neutrinos in the nuclear fusion processes inside the Sun's core. They have a special place in neutrino physics as they not only aid us in understanding our Sun and what powers it [1-3], but also help us in unraveling various neutrino properties such as neutrino oscillations and the effect of neutrino-matter interactions [4-9]. Moreover, neutrinos from the Sun enable us to put upper limits on the neutrino magnetic moment [10] and the accumulation of dark matter [11] inside the Sun.

At present, there are two principal detector types used for the measurement of solar neutrinos: Water Cherenkov (WCh) $[3,12,13]$ and Liquid Scintillator (LS) detectors [14-17]. They detect solar neutrinos via elastic scattering off electrons. The light produced by the recoil electrons is typically detected by photomultiplier tubes (PMTs). Both these detector types have their own sets of advantages and disadvantages. In WCh detectors, the Cherenkov light emission from the recoil electrons enables the directional reconstruction of the final-state lepton, that is essential for background suppression and particle identification based on the Cherenkov ring morphology $[3,18]$. The main disadvantage of WCh detection is a relatively small light yield at $\mathrm{MeV}$ energies, resulting in a higher energy threshold as well as poorer resolution compared to LS detectors. Only charged particles that have a velocity faster than the speed of light in the medium can be detected. This is determined by the refractive index $n$ of the medium. For water with $n \approx 1.33$, this results in a kinetic energy threshold of about $0.25 \mathrm{MeV}$ for electrons. In practice, the effective low energy threshold is higher due to the presence of radioactive background and PMT dark noise. Since the amount of Cherenkov light emitted close to the threshold is small, this low light yield makes it challenging to trigger an event and perform vertex and direction reconstruction. For example, taking into account the coverage, photon detection efficiency, and radioactive background, the lowest kinetic energy threshold for recoil electrons used in WCh detectors is $\sim 3.5 \mathrm{MeV}$, where only $\sim 30$ photoelectron hits are detected on average $[3,13]$.

On the other hand, large LS detectors have a relatively high light yield, and thus a higher energy resolution and a lower energy threshold, provided they have a sufficiently low level of residual radioactive contamination. The recoil electrons from solar neutrinos excite the liquid scintillator molecules, which produce isotropic scintillation light. For Borexino this corresponds to 500 photoelectron hits at $1 \mathrm{MeV}$ deposited energy detected with 2000 PMTs. This is equivalent to a 5\% energy resolution [19] and an effective low energy threshold of $\sim 0.19 \mathrm{MeV}$ for the spectroscopic analysis [20]. The main disadvantage of LS detectors is that the emitted Cherenkov photons are subdominant to such a degree that an event-by-event direction reconstruction has not been possible yet.

There is an ongoing effort for the development of hybrid detectors that could combine the advantages of both detector types, i.e., a low energy threshold, good energy resolution, and directional reconstruction using Cherenkov light [21]. This is motivated by the prospect of a rich physics program, ranging from the measurement of CNO solar neutrinos [22] to searches for neutrinoless double beta decay [23] for which solar neutrinos are a background. Moreover, the added scintillation signal provides a means to reconstruct hadronic recoils in the final states of $\mathrm{GeV}$ neutrino interactions, most relevant for future long-baseline oscillation experiments [21]. The research and development activities for these hybrid detectors can be summarized into four categories: (i) new target materials [24-26], (ii) fast photodetectors for time separation of Cherenkov and scintillation light [27], (iii) spectral sorting with band pass and dichroic filters [28,29], and (iv) new analysis techniques [30,31]. Only relatively small-scale experiments have been run so far, or are planned to run in the near future, such as CHESS [32], ANNIE [33], and FlatDot [34]. Contrariwise direction reconstruction with Cherenkov light in large-scale 
scintillator or hybrid neutrino detectors has only been studied with Monte Carlo simulations [21,22,35].

In this Letter, we present a measurement of sub-MeV solar neutrinos in the Borexino detector, using their associated Cherenkov light. For this, a specific energy region of interest has been selected using the recoil electron scintillation light signal. The measurement is performed through a novel analysis technique called "correlated and integrated directionality" (CID). This work provides an experimental proof of principle for the feasibility of using directional Cherenkov light in a monolithic, large-scale liquid scintillator detector. At the same time, the CID method also provides a robust, straightforward analysis technique that is readily applicable for other LS detectors like KamLAND [15], JUNO [17], and $\mathrm{SNO}+[16]$.

Borexino and solar neutrinos.-Borexino is a high lightyield LS detector whose main goal is the spectroscopic measurement of solar neutrinos [14]. The experiment is located at the Laboratori Nazionali del Gran Sasso (LNGS), Italy, at a depth of 3800 meters water equivalent to suppress the cosmic muon flux. The neutrino target at the detector center consists of $\sim 280$ tons of extremely radio-pure LS. It is composed of 1,2,4-trimethylbenzene (PC) solvent, mixed with 2,5-diphenyloxazole (PPO) as a fluor. The LS is contained in a thin nylon vessel, surrounded by two liquid buffer layers. The scintillator and buffers are contained in a stainless steel sphere, equipped with 2000 8-inch PMTs. This entire setup is enclosed in a water tank mounted with 200 PMTs serving as a muon veto. The experimental dataset is divided into three main phases: Phase I (May 2007-May 2010), phase II (December 2011-May 2016), and phase III (July 2016-October 2021). Borexino's ability to measure solar neutrinos through spectral fits has already been well demonstrated in the past years through the complete spectroscopy of $p p$-chain neutrinos $[1,36,37]$ and the first direct detection of $\mathrm{CNO}$ neutrinos [2]. The directionality measurement reported here is performed on individual photon hits of the events selected in a specific energy region of interest (ROI) to increase the signal-tobackground ratio. This facilitates the statistical separation of solar neutrinos and the intrinsic, isotropic radioactive background in the LS. The signal consists of electrons scattering off $0.862 \mathrm{MeV}$ monoenergetic ${ }^{7} \mathrm{Be}$ solar neutrinos ( $\sim 90 \%$ of signal), $1.44 \mathrm{MeV}$ monoenergetic pep solar neutrinos, and CNO solar neutrinos with an end point at $1.74 \mathrm{MeV}$. Solar neutrinos are named corresponding to the reaction process in which they are created. Since solar neutrinos are detected via their elastic scattering off electrons in the LS, even monoenergetic neutrinos show a continuous spectrum, characterized by a Compton-like edge. In the case of ${ }^{7} \mathrm{Be}$ solar neutrinos, this edge is at around $0.66 \mathrm{MeV}$ (see Fig. 1). The main backgrounds for this analysis include intrinsic radioactive $\beta^{-}$emitters, namely, ${ }^{210} \mathrm{Bi}(Q=1.162 \mathrm{MeV})$ and ${ }^{85} \mathrm{Kr}(Q=0.687 \mathrm{MeV})$.

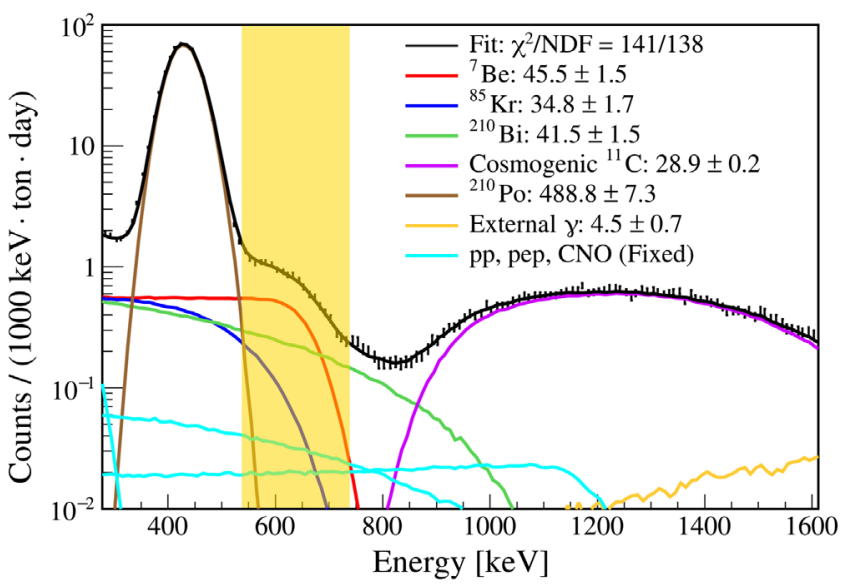

FIG. 1. The energy spectrum of phase I data (black points) along with the spectral fit (black line) performed using the PDFs of the different solar neutrino components and backgrounds [38]. The energy ROI used for the directional analysis of phase I is shown as a shaded yellow area.

Cherenkov light in Borexino.-Because of the wavelength dependence $\left(\lambda^{-2}\right)$ of the Cherenkov spectrum, most photons are produced in the ultraviolet (UV) region. These Cherenkov photons are absorbed by the fluor PPO and are subsequently reemitted as scintillation light. In Borexino, both Cherenkov and scintillation light spectra are detected above a wavelength of $370 \mathrm{~nm}$, where the PPO absorption becomes negligible. The spectra and velocity of Cherenkov and scintillation light in the LS depend on the wavelengthdependent refractive index (Fig. 9 in Ref. [39]). Considering $n \approx 1.55$ at $400 \mathrm{~nm}$, Cherenkov light is produced when the kinetic energy of the recoil electron from a solar neutrino exceeds $0.16 \mathrm{MeV}$. Cherenkov light is emitted at picosecond timescale while the fastest scintillation light component from the LS has an emission time constant at the nanosecond level. For this reason, the observed ratio of Cherenkov photons in the first few nanoseconds of a recorded event is considerably higher than for the entire event time. The detected PMT hit time distributions are broadened by various optical processes in the LS, the transit time spread (TTS) of the PMTs, jitter of the electronics, and precision of PMT time calibration. All the above-mentioned effects, as well as the multiple scattering of the recoil electrons in the LS, are taken into account in the customized GEANT- 4 based Monte Carlo (MC) simulation developed for Borexino [39]. The parameters of the MC have been tuned to reproduce the signals of radioactive sources used in the calibration campaign [19].

The distinct time behaviors of Cherenkov and scintillation photons are illustrated in Fig. 2. It shows the time-offlight corrected PMT hit time distributions obtained from $\mathrm{MC}$ for ${ }^{7} \mathrm{Be}$ solar neutrino recoil electrons in the ROI. In the chosen energy ROI, on average 270 PMT hits (normalized to 2000 live PMTs) are detected per event and MC predicts that only $\sim 1$ PMT hit per event is caused by 


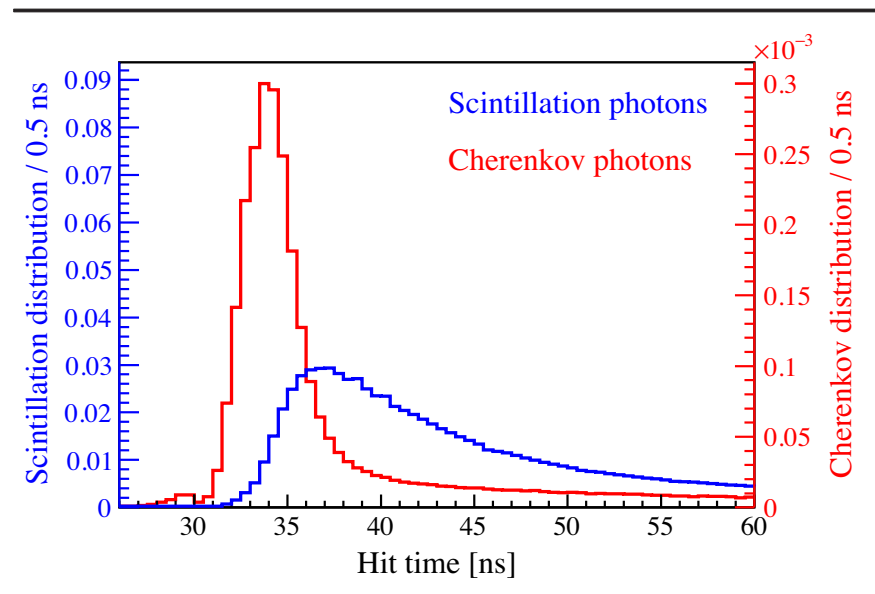

FIG. 2. Time-of-flight corrected hit times of ${ }^{7} \mathrm{Be}$ solar neutrino recoil electrons ( 0.54 to $0.74 \mathrm{MeV}$ ) as obtained from the Borexino MC. The left $y$ axis shows the scintillation light (blue), where the area is normalized to 1 . The Cherenkov light (red) is shown on the right $y$ axis and the area is normalized to the number of Cherenkov hits relative to scintillation $(\sim 0.4 \%)$. The scintillation light profile also includes photons that have been produced in the Cherenkov process, but have been absorbed and reemitted by the LS.

Cherenkov light. This highly disfavors an event-by-event directional reconstruction in Borexino.

Correlated and Integrated Directionality (CID). - In the CID technique, we correlate the detected PMT-hit pattern of a selected event to the well-known position of the Sun, and then integrate it for a large number of events. This results in an angular distribution between the hit PMTs and the solar direction which is illustrated in Fig. 3. Because of the event kinematics, the angular distribution of recoil electrons is centered around the direction of the incident solar neutrinos. The Cherenkov light is produced almost instantaneously and carries the directional information of the recoil electron, and thus approximately that of the solar neutrino. The dominant scintillation light is emitted isotropically and has no correlation to the Sun's position. Since events are detected in real time, the position of the Sun is well known for each event. The directional angle $\alpha$ is defined for each PMT hit as the angle between the known solar direction and the photon direction, given by the reconstructed event vertex of the recoil electron and the hit PMT position. Given the energy ROI and the refractive index of the LS, the CID distribution of solar neutrinos is expected to have a signature Cherenkov peak at $\cos \alpha \sim 0.7$ [see Fig. 4(b)]. Since the Cherenkov and scintillation light from the radioactive background inside Borexino has no correlation to the Sun's position, it instead produces a flat CID $\cos \alpha$ distribution. These CID distributions of solar neutrinos and background events can be disentangled by fitting MC-generated probability density functions (PDFs) for the directional signal and flat background to a highstatistics dataset. Note that the CID method on its own is largely insensitive to different kinds of solar neutrinos like ${ }^{7} \mathrm{Be}$, pep, and $\mathrm{CNO}$ in the chosen ROI.

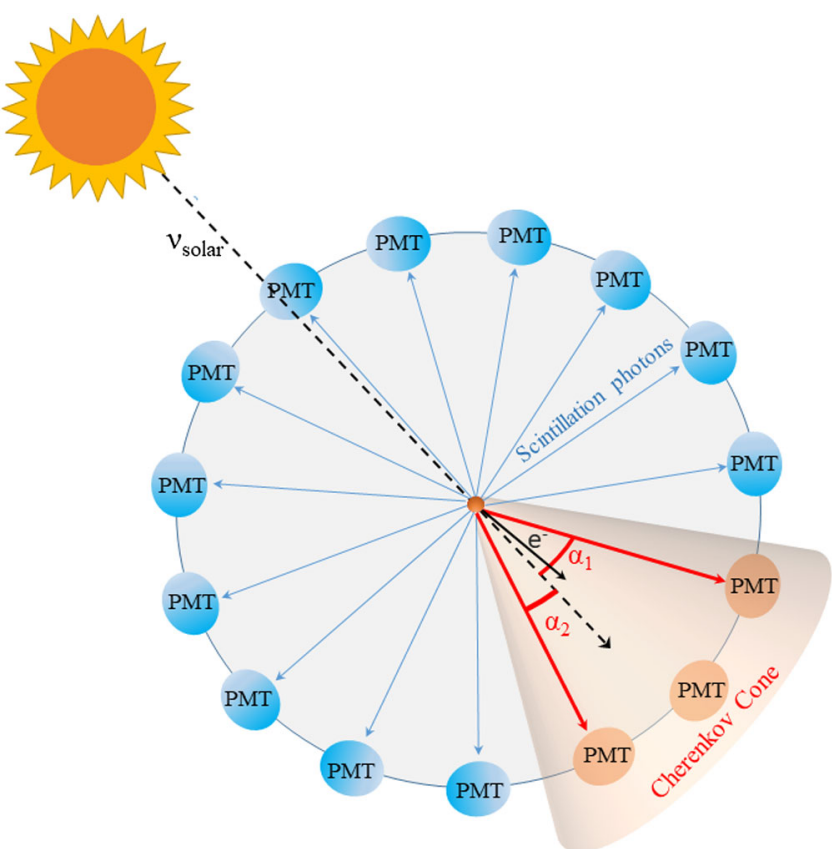

FIG. 3. Angular correlation of photon hits expressed in terms of the directional angle $\alpha$ given by the reconstructed vertex of the solar neutrino event and the position of the Sun. An electron recoiling off a solar neutrino in the detector produces isotropic scintillation light (blue arrows) uncorrelated to the Sun, as well as a Cherenkov cone (orange arrows) in the solar direction. In this example, the first two event hits are both Cherenkov photons and their respective directional angles are $\alpha_{1}$ and $\alpha_{2}$. The direction of Cherenkov and scintillation photons of radioactive background events are not correlated to the position of the Sun.

Analysis methods.-The principal dataset of this analysis consists of phase I. The data selection for this measurement follows a procedure similar to the standard low-energy solar neutrino analyses of Borexino [20,38]. However, the events are selected in a smaller energy ROI between 0.54-0.74 MeV (Fig. 1). In this ROI, external gammas originating from outside the LS are still negligible until a radius $r<3.3 \mathrm{~m}$, allowing us to use an enlarged fiducial volume of $132 \mathrm{t}$. In addition, pulse shape discrimination [41] is applied to remove $\alpha$ background from radioactive ${ }^{210} \mathrm{Po}(Q=5.304 \mathrm{MeV})$ in the scintillator. Its quenched energy spectrum (0.28-0.63 MeV electron equivalent) falls partially inside the ROI. In the chosen ROI, the overall ratio of Cherenkov to scintillation photons expected from $\mathrm{MC}$ is only $\sim 0.4 \%$. Unlike the usual event-based analyses of Borexino, the CID method is performed on the individual photon hits. As a consequence, we have adopted the " $N^{\text {th }}$ hit method": The times of the photon hits of each selected event are corrected with their time of flight between the reconstructed event vertex and the PMT that detected the hit. They are then sorted in time with respect to the reconstructed start time of the event. The analysis is restricted to the 1 st and 2 nd hits as they were found to have the highest Cherenkov ratio, capable of distinguishing 
the directional differences between signal and background in MC. For both 1st and 2nd hits separately, CID $\cos \alpha$ distributions are produced by summing over the hits of all selected events.
The number of solar neutrinos $N_{\text {solar- } \nu}$ is extracted through a $\chi^{2}$ fit of the CID $\cos \alpha$ data spectrum to the MC PDFs of the solar neutrino signal and $\beta^{-}$background. The test statistics is defined as follows:

$$
\chi^{2}\left(N_{\text {solar }-\nu}\right)=\sum_{n=1}^{N} \sum_{i=1}^{I}\left(\frac{\left((\cos \alpha)_{n, i}^{D}-(\cos \alpha)_{n, i}^{M}\left(N_{\text {solar }-\nu}, \Delta r_{\mathrm{dir}}, g v_{\mathrm{ch}}^{\mathrm{corr}}\right)\right)^{2}}{\left(\sigma_{n, i}^{D}\right)^{2}+\left(\sigma_{n, i}^{M}\right)^{2}}+\frac{\left(g v_{\mathrm{ch}}^{\text {corr }}-0.108 \mathrm{~ns} \mathrm{~m}^{-1}\right)^{2}}{\left(0.039 \mathrm{~ns} \mathrm{~m}^{-1}\right)^{2}}\right),
$$

with the hit index $n=1,2$ and the angular index $i$ from 1 to the total number of bins $I=60$ in the range $-1<\cos \alpha<+1 .(\cos \alpha)_{n, i}^{D}$ and $(\cos \alpha)_{n, i}^{M}$ are the $\cos \alpha$ values for the ith bin of the nth hit of data and MC, respectively, and, $\sigma_{n, i}^{D}$ and $\sigma_{n, i}^{M}$ are their corresponding statistical errors. The parameters $\Delta r_{\text {dir }}$ and $g v_{\mathrm{ch}}^{\mathrm{corr}}$ are sufficient to parametrize the differences between data and MC. Their values are small and have no impact on the eventbased algorithms of Borexino. The parameter $\Delta r_{\text {dir }}$ takes into account a systematic shift in the reconstructed vertex position of the recoil electron with respect to the initial electron direction, which is correlated to the solar neutrino direction. It is not visible on an event-by-event basis, but is observed in MC and has an influence on the summed CID $\cos \alpha$ distribution of solar neutrinos. The value of $\Delta r_{\text {dir }}$ in data cannot be determined by Borexino calibrations and hence is left free to vary in the fit. The parameter $g v_{\mathrm{ch}}^{\text {corr }}$ is an effective correction of the group velocity for Cherenkov photons relative to scintillation photons and is treated as a nuisance parameter with a Gaussian pull-term in the fit, based on gamma calibration data as explained below. This correction term reflects the remaining uncertainty on the effective Cherenkov wavelength spectrum and the wavelength-dependent refractive index implemented in the detector MC. These can affect the relative group velocities of scintillation and Cherenkov photons and thus change their effective ratio for the first few photon hits used in the analysis.

We have performed a calibration of the effective Cherenkov group velocity correction using the available gamma calibration sources from Borexino's 2009 calibration campaign [19]. Because of the known positions of the gamma sources from CCD cameras, the initial directions of the gamma rays can be reconstructed. The Cherenkov photons from the Compton-scattered electrons can be correlated to the reconstructed gamma direction similar to the CID analysis. This calibration results in a value of $g v_{\mathrm{ch}}^{\text {corr }}=(0.108 \pm 0.039) \mathrm{ns} \mathrm{m}^{-1}$. Since the calibration has been performed at the end of phase I data taking and subnanosecond stability of effective PMT timing cannot be guaranteed for long time periods, this measurement is considered valid only for phase I. Note that Cherenkov photons from background PDFs are not influenced by $\Delta r_{\text {dir }}$ and $g v_{\mathrm{ch}}^{\text {corr }}$ as the direction of both Cherenkov and scintillation light are uncorrelated to the position of the Sun. Both $\Delta r_{\text {dir }}$ and $g v_{\mathrm{ch}}^{\text {corr }}$ are explained in more detail in the companion paper [42]. Note that in future LS detectors, the uncertainties arising from both parameters might be substantially reduced by the deployment of a dedicated electron Cherenkov source.

Further systematic effects have been investigated: the choice of the histogram binning, the number of early PMT $N$ th hits, and the influence of the number and distribution of active PMTs. In total, they contribute a relative systematic uncertainty of $8.7 \%$ on the $N_{\text {solar- } \nu}$ result. Other sources of uncertainty, such as the effect of a nonuniform distribution of radioactive background in the fiducial volume have been studied, but found to be negligible (more details in the companion paper).

Results.-Figure 4(a) shows the $\Delta \chi^{2}$ between data and the best fit as a function of the number of solar neutrino events $N_{\text {solar- } \nu}$. We show the $\Delta \chi^{2}$ profile both with and without the systematic uncertainty of $8.7 \%$. The agreement between the best fit and data is given by $\chi^{2} / n d f=$ 124.6/117 ( $p$-value $=0.30)$, based on the histograms of the first two PMT hits of all events, with 60 bins each. The resulting best fit for the number of solar neutrino events is $N_{\text {solar- } \nu}=10887_{-2103}^{+2386}$ (stat) \pm 947 (syst) $(68 \%$ C.I.) out of the 19904 selected events, and consists of ${ }^{7} \mathrm{Be}$, pep, and $\mathrm{CNO}$ neutrinos. Figure 4(a) also shows the $68 \%$ confidence intervals for the measured $N_{\text {solar- } \nu}$ and the expected value according to the standard solar model (SSM) [40,43], represented by the blue and orange bands, respectively. The SSM has varied predictions on the so-called "metallicity," i.e., the amount of metals heavier than ${ }^{4} \mathrm{He}$ in the Sun. Since this affects the expected number of solar neutrinos in Borexino, the difference between the low and high metallicity model predictions are included as a systematic uncertainty. The measured number of solar neutrinos is well in agreement with the SSM expectation of $N_{\mathrm{SSM}}=10187_{-1127}^{+541}$. The background-only hypothesis can be excluded with $\Delta \chi^{2}>25$, which corresponds to a $>5 \sigma$ detection of sub-MeV solar neutrinos using the CID method. Figure 4(b) shows the measured $\cos \alpha$ distribution for the first PMT hits of the data events together with the best fit and the neutrino-only and background-only $\cos \alpha$ distributions. For illustration purposes, this is shown for 10 bins instead of the 60 bins used in the final fit. 


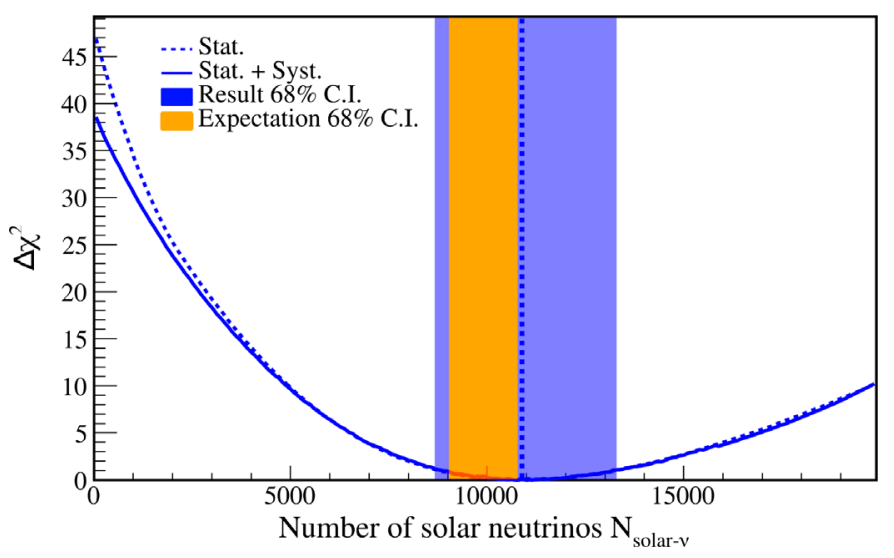

(a)

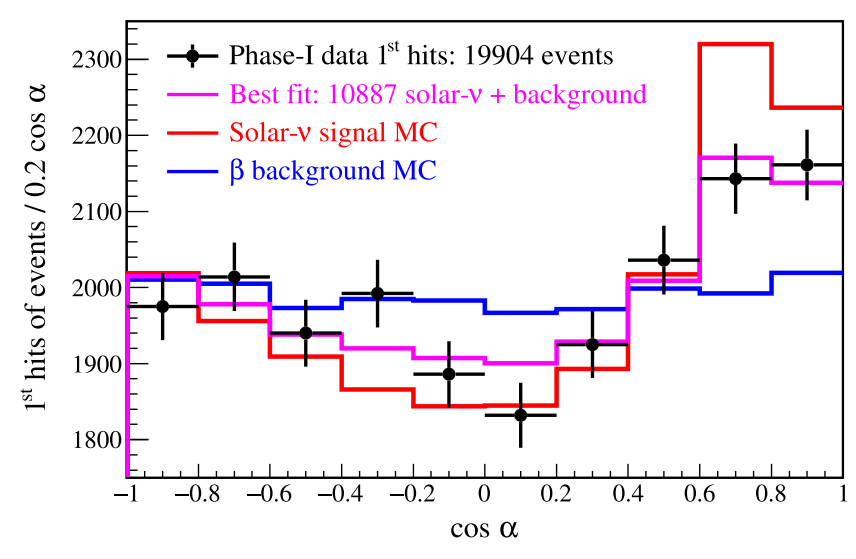

(b)

FIG. 4. (a) The sum of the $\Delta \chi^{2}$ profiles of the 1st and 2nd hits from the fit as a function of the observed number of solar neutrinos $N_{\text {solar- } \nu}$, with (blue solid curve) and without (blue dotted curve) the systematic uncertainty. The no-neutrino signal hypothesis $N_{\text {solar- } \nu}=0$, can be rejected with $\Delta \chi^{2}>25,>5 \sigma$ C.L. The $68 \%$ C.I. (blue shaded band) from the $\Delta \chi^{2}$ profile gives $N_{\text {solar- } \nu}=10887_{-2103}^{+2386}$ (stat) \pm 947 (syst). The best fit gives a $\chi^{2} / n d f=124.6 / 117$. The $68 \%$ C.I. of the expected solar neutrino signal based on the SSM predictions [40] is shown as an orange band. (b) The cos $\alpha$ distributions of the 1 st hits of all the selected events (black points) compared with the best fit curve (magenta) of the resulting number of solar neutrinos plus background. The MC PDFs of pure neutrino signal (red) and $\beta$ background (blue) used in the fit are shown as well, normalized to the same 19904 events. For illustration purposes, the histograms are shown with 10 bins instead of the 60 bins as used in the final fit.

Using the CID measurement of $N_{\text {solar- } \nu}$, the ${ }^{7} \mathrm{Be}$ interaction rate $\mathrm{R}\left({ }^{7} \mathrm{Be}\right)_{\mathrm{CID}}$ has been calculated as $\mathrm{R}\left({ }^{7} \mathrm{Be}\right)_{\mathrm{CID}}=$ $51.6_{-12.5}^{+13.9} \mathrm{cpd} / 100 \mathrm{t}$ for the full ${ }^{7} \mathrm{Be}$ neutrino energy $(0.384$ and $0.862 \mathrm{MeV}$ monoenergetic lines). For this the pep and CNO neutrino rates have been fixed to their SSM predictions [43]. The small errors arising from pep and CNO neutrino predictions are included in the systematic uncertainty (refer companion paper). This ${ }^{7} \mathrm{Be}$ rate is well in agreement with the results of the phase I spectroscopy $\mathrm{R}\left({ }^{7} \mathrm{Be}\right)=47.9 \pm 2.3 \mathrm{cpd} / 100 \mathrm{t}$ where the pep and CNO neutrino rates were fixed to their SSM predictions as well [38].

Conclusions.-For the first time, we have measured sub$\mathrm{MeV}$ solar neutrinos using their directional Cherenkov light, through the CID method in a traditional, large-scale LS detector. While this measurement on its own features relatively large statistical and systematic uncertainties, it still provides experimental proof that the directional information of Cherenkov light is accessible even for sub-MeV neutrinos in a high light-yield LS medium. In future analyses the CID measurement could be combined with a standard spectral fit, and thus help to disentangle neutrino signal and backgrounds. This might be interesting especially if there is a degeneracy of signal and background energy spectra, as is the case for the ${ }^{210} \mathrm{Bi}$ background and CNO solar neutrinos [2] in Borexino.

We acknowledge the generous hospitality and support of the Laboratori Nazionali del Gran Sasso (Italy). The Borexino program is made possible by funding from Istituto Nazionale di Fisica Nucleare (INFN) (Italy),
National Science Foundation (NSF) (USA), Deutsche Forschungsgemeinschaft (DFG), Cluster of Excellence PRISMA+ (Project ID 390831469), and recruitment initiative of Helmholtz-Gemeinschaft (HGF) (Germany), Russian Foundation for Basic Research (RFBR) (Grants No. 19-02-00097A) and Russian Science Foundation (RSF) (Grant No. 21-12-00063) (Russia), and Narodowe Centrum Nauki (NCN) (Grant No. UMO 2017/26/M/ST2/ 00915) (Poland). We gratefully acknowledge the computing services of the Bologna INFN-CNAF data centre and U-Lite Computing Center and Network Service at LNGS (Italy).

*Present address: University of California, Berkeley, Department of Physics, California 94720, Berkeley, USA.

†Present address: Dipartimento di Fisica, Università degli Studi Federico II e INFN, 80126 Napoli, Italy.

*Present address: Universidad Autónoma de Madrid, Ciudad Universitaria de Cantoblanco, 28049 Madrid, Spain.

${ }^{\S}$ Present address: INFN Laboratori Nazionali del Gran Sasso, 67010 Assergi (AQ), Italy.

"Present address: Dipartimento di Fisica e Astronomia dell'Università di Padova and INFN Sezione di Padova, 35131 Padova, Italy.

TPresent address: Istituto Superiore per la Protezione e la Ricerca Ambientale, 00144 Roma, Italy.

*Borexino Collaboration Spokesperson. borex@lngs.infn.it

[1] M. Agostini et al. (Borexino Collaboration), Nature (London) 562, 505 (2018). 
[2] M. Agostini et al. (Borexino Collaboration), Nature (London) 587, 577 (2020).

[3] K. Abe et al. (Super-Kamiokande Collaboration), Phys. Rev. D 94, 052010 (2016).

[4] B. T. Cleveland, T. Daily, R. Davis, Jr., J. R. Distel, K. Lande, C. K. Lee, P. S. Wildenhain, and J. Ullman, Astrophys. J. 496, 505 (1998).

[5] W. Hampel et al. (GALLEX Collaboration), Phys. Lett. B 447, 127 (1999).

[6] J. N. Abdurashitov, V. N. Gavrin, V. V. Gorbachev, P. P. Gurkina, T. V. Ibragimova et al. (SAGE Collaboration), Phys. Rev. C 80, 015807 (2009).

[7] Q. R. Ahmad et al. (SNO Collaboration), Phys. Rev. Lett. 87, 071301 (2001).

[8] S. Fukuda et al. (Super-Kamiokande Collaboration), Phys. Rev. Lett. 86, 5656 (2001).

[9] A. Friedland, C. Lunardini, and C. Peña-Garay, Phys. Lett. B 594, 347 (2004).

[10] M. Agostini et al. (Borexino Collaboration), Phys. Rev. D 96, 091103 (2017).

[11] K. Choi et al. (Super-Kamiokande Collaboration), Phys. Rev. Lett. 114, 141301 (2015).

[12] M. Anderson et al. (SNO+ Collaboration), Phys. Rev. D 99, 012012 (2019).

[13] B. Aharmim et al. (SNO Collaboration), Phys. Rev. C 81, 055504 (2010).

[14] G. Alimonti et al. (Borexino Collaboration), Nucl. Instrum. Methods Phys. Res., Sect. A 600, 568 (2009).

[15] A. Gando et al. (KamLAND Collaboration), Phys. Rev. C 92, 055808 (2015).

[16] M. Anderson et al. (SNO+ Collaboration), J. Instrum. 16, P05009 (2021).

[17] F. An et al. (JUNO Collaboration), J. Phys. G 43, 030401 (2016).

[18] M. Jiang et al. (Super-Kamiokande Collaboration), Prog. Theor. Exp. Phys. 2019 (2019).

[19] H. Back et al. (Borexino Collaboration), J. Instrum. 7, P10018 (2012).

[20] M. Agostini et al. (Borexino Collaboration), Phys. Rev. D 100, 082004 (2019).

[21] M. Askins et al., Eur. Phys. J. C 80, 416 (2020).

[22] R. Bonventre and G. D. Orebi Gann, Eur. Phys. J. C 78, 435 (2018).

[23] C. Aberle, A. Elagin, H. J. Frisch, M. Wetstein, and L. Winslow, J. Instrum. 9, P06012 (2014).
[24] M. Yeh, S. Hans, W. Beriguete, R. Rosero, L. Hu, R. L. Hahn, M. V. Diwan, D. E. Jaffe, S. H. Kettell, and L. Littenberg, Nucl. Instrum. Methods Phys. Res., Sect. A 660, 51 (2011).

[25] Z. Guo, M. Yeh, R. Zhang, D.-W. Cao, M. Qi, Z. Wang, and S. Chen, Astropart. Phys. 109, 33 (2019).

[26] E. Graham, D. Gooding, J. Gruszko, C. Grant, B. Naranjo, and L. Winslow, J. Instrum. 14, P11024 (2019).

[27] A. Lyashenko et al., Nucl. Instrum. Methods Phys. Res., Sect. A 732, 190 (2013).

[28] T. Kaptanoglu, M. Luo, B. Land, A. Bacon, and J. R. Klein, Phys. Rev. D 101, 072002 (2020).

[29] T. Kaptanoglu, M. Luo, and J. Klein, J. Instrum. 14, T05001 (2019).

[30] B. Wonsak, C. I. Hagner, D. A. Hellgartner, K. Loo, S. Lorenz, D. J. Meyhöfer, L. Oberauer, H. Rebber, W. H. Trzaska, and M. Wurm, J. Instrum. 13, P07005 (2018).

[31] A. Elagin, H. J. Frisch, B. Naranjo, J. Ouellet, L. Winslow, and T. Wongjirad, Nucl. Instrum. Methods Phys. Res., Sect. A 849, 102 (2017).

[32] J. Caravaca, F. B. Descamps, B. J. Land, M. Yeh, and G. D. Orebi Gann, Eur. Phys. J. C 77, 811 (2017).

[33] A. R. Back et al. (ANNIE Collaboration), arXiv:1707.08222.

[34] J. Gruszko, B. Naranjo, B. Daniel, A. Elagin, D. Gooding, C. Grant, J. Ouellet, and L. Winslow, J. Instrum. 14, P02005 (2019).

[35] B. J. Land, Z. Bagdasarian, J. Caravaca, M. Smiley, M. Yeh, and G. D. OrebiGann, Phys. Rev. D 103, 052004 (2021).

[36] G. Bellini et al. (Borexino Collaboration), Phys. Rev. Lett. 107, 141302 (2011).

[37] G. Bellini et al. (Borexino Collaboration), Phys. Rev. Lett. 108, 051302 (2012).

[38] G. Bellini et al. (Borexino Collaboration), Phys. Rev. D 89, 112007 (2014).

[39] M. Agostini et al. (Borexino Collaboration), Astropart. Phys. 97, 136 (2018).

[40] M. Agostini et al. (Borexino Collaboration), Eur. Phys. J. C 80, 1091 (2020).

[41] M. Agostini et al. (Borexino Collaboration), Phys. Rev. D 101, 012009 (2020).

[42] M. Agostini et al. (Borexino Collaboration), companion paper, Phys. Rev. D 105, 052002 (2022).

[43] N. Vinyoles,A. M. Serenelli, F. L. Villante, S. Basu, J. Bergström, M. C. Gonzalez-Garcia, M. Maltoni, C. PeñaGaray, and N. Song, Astrophys. J. 835, 202 (2017). 